\title{
Infrared Image Segmentation for Power Equipment Using Linear Spectral Clustering and Maximal Similarity-based Region Merging
}

\author{
Haiyan $\mathrm{Yu}^{1}$, Jihong $\mathrm{Wang}^{2^{*}}$ \\ ${ }^{1}$ School of Information Engineering, Zhengzhou University of Science and Technology, Zhengzhou, China \\ ${ }^{2}$ School of Electrical Engineering, Zhengzhou University of Science and Technology, Zhengzhou, China \\ zkdywjh@126.com, aqiufenga@163.com \\ Received 27 September 2021; Revised 15 October 2021; Accepted 27 October 2021
}

\begin{abstract}
The diagnostic method of power equipment based on infrared images is widely used because it has the advantages of non-contact and does not affect the online operation of power equipment. However, in actual using, the power equipment diagnosis method based on infrared image still relies on manual judgment, that is, the detection personnel can judge the fault according to the obtained infrared image of power equipment by experience. This process consumes a lot of time, and the subjectivity is strong, misjudgment rate is higher, which cannot meet the requirements of modern smart grid development. Infrared image of power equipment contains a lot of noise, and the edge is fuzzy. In this paper, we propose a new infrared image segmentation method for power equipment by using linear spectral clustering and maximal similarity-based region merging under complex backgrounds. In this method, the linear spectral clustering algorithm (LSC) is used to segment the image into super-pixels, and the pixels with similar color and distance are clustered to the same center. The calculated OTSU threshold based on the global image is used to pre-label the background of each superpixel block. The maximum similarity-based region merging algorithm (MSRM) is utilized to merge the superpixel blocks. Meanwhile, it obtains the target equipment, the over-segmentation and under-segmentation rates are reduced effectively. Finally, the mathematical morphology algorithm is used to post-process the image. Experimental results show that, compared with other algorithms, this new method can obtain more accurate and complete target equipment under complex background.
\end{abstract}

Keywords: Infrared image segmentation, power equipment, linear spectral clustering, maximal similaritybased region merging, OTSU threshold, mathematical morphology operation

\section{Introduction}

For the maintenance of power equipment, it is a widely used and effective method to use infrared imaging instruments to diagnose the fault of the equipment to be tested [1]. By processing the infrared image acquired on-site, the fault state of the equipment is diagnosed and the fault area of the equipment is determined. At present, the infrared image processing of power equipment is mainly divided into two categories:

hot spot segmentation. This segmentation method mainly uses the gray level information, color information and edge information of the image to find the heating part of the fault equipment directly. Reference [2] used the one-dimensional OTSU algorithm to segment the seed region and used the region growing algorithm to segment the fault region of the power equipment. Reference [3] extracted the fault region of infrared image of power equipment by simplifying the internal parameters of the pulse-coupled neural network and combining the local features in the boundary between the non-fault region and fault region neighborhood. Turbopixel superpixel segmentation algorithm was used in reference [4] to segment infrared fault images, and the fault areas were extracted with chromaticity information.

the overall segmentation of electric equipment. This segmentation method mainly uses the color, texture and other information of the equipment to segment the whole equipment, and provides basic images for the subsequent judgments such as thermal fault status, equipment damage detection, foreign body inclusion diagnosis and other issues. Reference [5] used K-means algorithm and Fast-match algorithm to locate the target in visible images, and used the approximate affine transformation of infrared image and visible image to segment the target equipment. In reference [6], the information points were clustered according to the minimum distance principle in the Lab color space, and then the density similarity factor was used to filter out the image noise and complete the infrared image target segmentation of power equipment. Reference [7] used prior information, gamma transform and Retinex algorithm to enhance image contrast, and used multi-scale smoothing filter to filter most background 
interference in infrared images of power equipment. Reference [8] improved the membership degree of Fuzzy C-means (FCM) algorithm by simplifying data set, optimizing clustering and using Gaussian function to complete the segmentation of power equipment.

The hot spot is segmented to find the fault area of the equipment, which is applicable to the image where the fault of the equipment has been judged. However, the normal state of the equipment and the pre-fault diagnosis can not be made. The power equipment is segmented as a whole. After the segmentation is completed, the fault state of the equipment is diagnosed by judging the temperature relationship and gray level relationship between each structural area of the equipment. It can make a clear judgment on whether the equipment is in fault and the fault level. However, in the process of infrared imaging, the image contrast is often low due to the complex and changeable environment, large background interference and other problems [9]. In the process of thermal fault diagnosis of power equipment, it needs to spend a lot of energy to obtain the whole target and fault area, so as to get accurate diagnosis results. Therefore, the segmentation of the target subject is the key to improve the efficiency and accuracy of thermal fault diagnosis of power equipment.

Taking the global infrared image of power equipment as the research object and combining it with the characteristics of the infrared image of power equipment, this paper proposes a linear spectral clustering algorithm (LSC) combining Maximal Similarity-Based Region Merging (MSRM) and OTSU algorithm for power equipment image segmentation in complex background. First, LSC algorithm is used to segment the whole image. Then the OTSU algorithm is used to calculate the global threshold, and the threshold is used to pre-label the background of the segmented super-pixel blocks. Then the MSRM algorithm merges the super-pixel blocks to obtain the rough extraction of the target device. Finally, mathematical morphology algorithm is used to post-process the segmented image to achieve the overall segmentation of the target device under complex background.

\section{Proposed Infrared Image Segmentation for Power Equipment}

\subsection{LSC Super-pixel Segmentation}

In the field of image segmentation, it is a common method to cluster the pixel points with similar color, brightness or texture into the same super-pixel block by using graph theory and clustering theory. At present, normalized cut (NCuts) [10], weighted k-means [11], simple linear iterative clustering (SLIC) [12] and other algorithms are commonly used. However, these algorithms have high computational complexity, they ignore the relationship between local and global images, and fail to make full use of small image feature information. The segmented super-pixel block boundary has a poor fit with the natural boundary of the object. Linear Spectral Clustering (LSC) algorithm adopted in this paper was proposed in 2017. Compared with other algorithms, this algorithm has a greater improvement in computational complexity, computational efficiency and the quality of generating super-pixel blocks [13].

LSC algorithm is a linear iterative super-pixel segmentation algorithm based on the cost function study of the relationship between NCuts and the weighted k-means. In the high-dimensional feature space, when the similarity between two points is equal to the weighted inner product between the corresponding vectors, then the cost function of NCuts and weighted k-means is equivalent. The cost function of weighted k-means is shown in equation (1):

$$
\begin{gathered}
F_{k m}=\sum_{k=1}^{K} \sum_{p \in \pi_{k}} w(p)\left\|\phi(p)-m_{k}\right\|^{2} . \\
m_{k}=\frac{\sum_{q \in \pi_{k}} w(q) \phi(q)}{\sum_{q \in \pi_{k}} w(q)} .
\end{gathered}
$$

The cost function of NCuts is shown in equation (3): 


$$
F_{\text {Ncuts }}=\frac{1}{K} \sum_{k=1}^{K} \frac{\sum_{p \in \pi_{k}} \sum_{q \in \pi_{k}} w(p, q)}{\sum_{p \in \pi_{k}} \sum_{q \in V} w(p, q)} .
$$

Where $p$ and $q$ are the two different points of the image. $w(p)$ is the weight of point $p . \mathrm{K}$ is the number of clusters. $\pi_{k}$ is the k-th class. $\phi(p)$ is the function of point $p$ mapped to the high eigenspace to improve the linear separability. $w(p, q)$ is the similarity of $p$ and $q . \mathrm{V}$ is the set of all points in the graph.

After introducing kernel matrix in NCuts and weighted k-means, the optimization problems of $F_{k m}$ and $F_{N c u t s}$ are transformed into matrix trajectory maximization problems, which enhances the connection between NCuts and weighted k-means. However, only if the kernel matrix is positive definite, its convergence can be guaranteed. Satisfying this condition requires the kernel function to be transformed. According to the sufficient and necessary conditions of the positive definite of Gram matrix, when equations (4) and (5) are both satisfied, the cost function optimization of NCuts and weighted k-means is mathematically equivalent, that is, the positive definite of the kernel matrix is satisfied:

$$
\begin{gathered}
w(p) \phi(p) \cdot w(q) \phi(q)=w(p, q) \forall p, q \in V . \\
w(p)=\sum_{q \in V} W(p, q), \forall p \in V .
\end{gathered}
$$

By substituting equations (4) and (5) into equations (1) and (2), we can get the equation:

$$
F_{k m}=C-\sum_{k=1}^{K} \frac{\sum_{p} \sum_{q} W(p, q)}{\sum_{p} \sum_{q \in V} W(p, q)}=C-K \times F_{\text {Ncuts }}
$$

In equation (6), $\mathrm{C}$ is a constant. The minimum value of $F_{k m}$ in the high-dimensional feature space is equivalent to the maximum value of $F_{\text {Ncuts }}$, that is, in the high-dimensional feature space defined by $\phi(\cdot)$, the optimal segmentation result obtained by NCuts algorithm is equal to the optimal segmentation result of weighted k-means.

\subsection{MSRM Super-pixel Merging}

After LSC super-pixel segmentation, the image is divided into several small regions. To achieve a complete segmentation of the target, the super-pixels forming the target region need to be merged, but this process requires some descriptors to distinguish the target region from the background region, such as color, edge, texture, shape and size etc,. For general images, color is an effective description of target features, which is more robust than other feature descriptors. According to the on-site detection requirements of power equipment and the characteristics of infrared images, there is a difference in color between the target equipment and the surrounding environment, especially when the target equipment is in the fault state, the difference will be more obvious.

On the basis of super-pixel segmentation, Maximal Similarity-Based Region Merging (MSRM) algorithm [14] takes advantage of the high similarity in color of the same object to segment the target. The color features of the region are described by color histogram. Sixteen quantization levels of color values are adopted for pixel points in the R, G and B color channels, and 163 colors are quantized in the color histogram. The algorithm takes the Babbitt distance as the similarity measure, and uses a merging mechanism based on the adaptive maximum similarity to identify all the unmarked regions under the guidance of labeled target and background. 
The merging rule of MSRM algorithm is as follows: let $\mathrm{Q}$ be the adjacent region of $\mathrm{R}$. $\bar{S}_{\mathrm{Q}}=\left\{S_{i}^{Q}\right\}(i=1, \ldots, q)$ is the adjacent region set of $\mathrm{Q}$. Then $\mathrm{R}$ is a subset of $\mathrm{S}$, and the similarity $\rho\left(Q, S_{i}^{Q}\right)$ between $\mathrm{Q}$ and all adjacent regions is calculated. If the similarity between neighborhood $\mathrm{R}$ and $\mathrm{Q}$ is the largest, then $\mathrm{R}$ and $\mathrm{Q}$ are merged, that is:

$$
\text { merge } R \text { and } Q \text {, if } \rho(R, Q)=\max _{i=1, \cdots, q} \rho\left(Q, S_{i}^{Q}\right)
$$

The Babbitt distance is:

$$
\rho(R, Q)=\sum_{u=1}^{4096} \sqrt{\text { Hist }_{R}^{u} \cdot \text { Hist }_{Q}^{u}}
$$

Where Hist $t_{R}^{u}$ is the probability of the color $u$ occurring in the region R.

\subsection{OTSU Threshold Calculation}

OTSU algorithm [15] is called maximum inter-class variance method. The basic principle is that images with gray level range $[0, \mathrm{~L}-1]$ are divided into background $C_{B}\left(C_{B}=1, \cdots, t\right)$ and foreground $C_{F}\left(C_{F}=t+1, \cdots, L-1\right)$ with threshold $t$ based on gray level information. When the inter-class variance of the two classes is the biggest, the calculated threshold $t$ is the optimal threshold T, namely:

$$
T=\operatorname{Arg} \max _{0 \leq t \leq L-1}\left\{P_{F} P_{B}\left(\mu_{F}-\mu_{B}\right)^{2}\right\} .
$$

Where $P_{F}$ and $P_{B}$ are the probabilities of foreground and background respectively. $\mu_{F}$ and $\mu_{B}$ are variances of foreground and background respectively.

The OTSU threshold has good robustness to the noise generated by molecular thermal motion. In image processing, most background areas with atmospheric environment can be filtered out.

\subsection{Morphological Open and Close Operation}

The mathematical morphology algorithm [16] uses the morphology operator to filter out the interference and retain the original information in the image, so as to obtain the smoother edge information and achieve the purpose of image analysis. The morphological open operation is that the structural element B first conducts the corrosion operation and then the expansion operation on set A. The operation smoothes out the contours of objects, weakens narrow parts and removes delicate protrusions. Its definition is as follows:

$$
A \circ B=(A \Theta B) \oplus B
$$

Morphological close operation is that the structural element B first conducts the expansion operation and then the corrosion operation on the set A. The algorithm fuses narrow notches with slender bends, eliminating smaller holes to fill in the gaps in the contour. Its definition is as follows:

$$
A \cdot B=(A \oplus B) \Theta B
$$

\subsection{The Flow Chart of the Proposed Algorithm}


The LSC super-pixel segmentation algorithm takes into account the edge information and color information of the image, and the obtained segmentation line has a higher fit degree and accuracy with the target. The MSRM super-pixel merging algorithm uses color as a descriptor and Babbitt distance as a similarity measure to enhance the robustness against noise and some small changes. The calculated threshold value by the OTSU algorithm has a good anti-noise ability to the atmospheric noise, which can remove the area in the infrared image that exists inside the target but should belong to the background. It makes up for the under-segmentation that may be caused by the MSRM super-pixel merging algorithm in the multi-connected region merging. Morphological open-close operation can filter out the fine protrusion and fill the gap of the contour, which is helpful to improve the segmentation accuracy.

However, the MSRM super-pixel merging algorithm generally marks the typical background block and target block in the segmentation process to guide the correct merging of super-pixel blocks. Therefore, combining with the characteristics of infrared images of power equipment, on-site detection requirements and existing problems in infrared image detection, the following marking rules are proposed:

\section{(A) Background Marking.}

Infrared image backgrounds of power equipment mainly have two categories: one is natural substances (e.g. air, trees, etc,.). Compared with electric equipment, this kind of background shows less heat in the image, that is, it has higher contrast with the target equipment. The other is other structures that are not related to the target equipment. This kind of background is a heating element like the target device. The constituent materials (such as concrete, etc.) have relatively large specific heat capacity and high reflectivity, which presents a low contrast with the target device in the infrared image. Therefore, the background marking rule is:

First, marking the super-pixel block with the lowest brightness in the image as the background block;

Second, marking the super-pixel block with higher brightness in the left and right edges of the image as the background block.

\section{(B) Target Marking.}

All the target devices are located in the center of the image, and the distance between the target device and the detection instrument is closer than the background device or object in the imaging process, so the temperature loss is small and the brightness of the image is higher. Therefore, the rule of target marking is that: marking the super-pixel block with the highest brightness in the image and not located at the edge of the image as the target.

The flow chart of the proposed image segmentation algorithm is shown in Fig. 1.

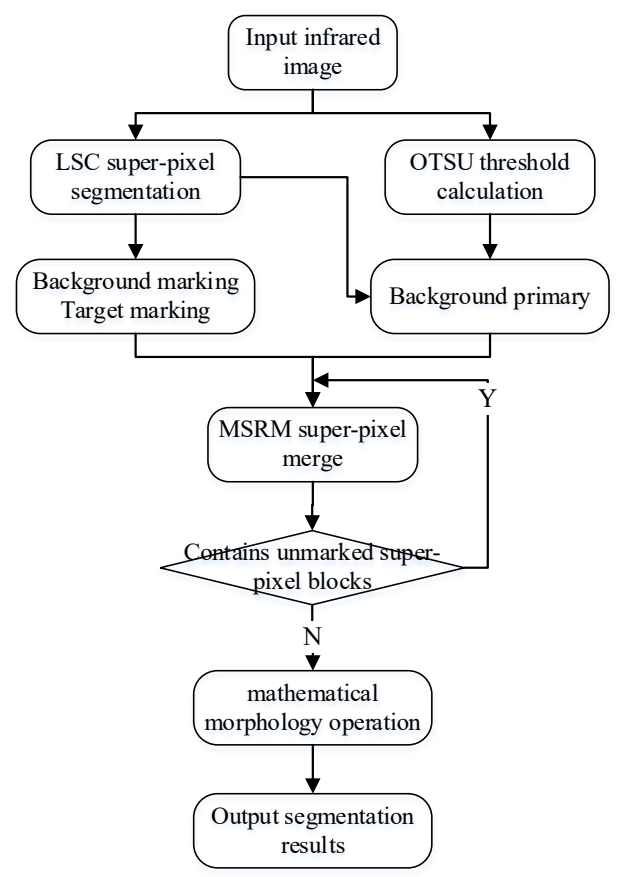

Fig. 1. The structure of the proposed segmentation method 
The proposed image segmentation algorithm in this paper is divided into four steps:

LSC super-pixel segmentation. Set the appropriate clustering value K, and divide the input infrared image into $\mathrm{K}$ super-pixel blocks.

Calculate OTSU threshold and pre-select background. The input infrared image is converted into a grayscale image and the global threshold $\mathrm{T}$ is calculated. On the basis of Step (1), the grayscale mean $G_{\text {avg }}(i=1, \cdots, K)$ of each super-pixel block is calculated. When $G_{\text {avg }}$ is less than the threshold T, the super-pixel block is recorded as the pre-selected block of the background.

MSRM super-pixel merging. The target block and the background block are marked according to the setting rules. At this time, the super-pixel block will be divided into three categories, namely the target marked block, the background marked block and the unmarked block.

Morphology open and close operation post-processing. The appropriate structural element B is selected, and the morphology open/close operation is used to perform post-processing on the target segmentation image obtained in Step 3, so as to eliminate the over-segmentation or under-segmentation problems caused by improper super-pixel segmentation.

\section{Experiments and Analysis}

The experiment environment is Windows 10, MATLAB+python, Intel(R) Core(TM) i7-6700 CPU @ 3.40GHz $3.41 \mathrm{GHz}, \mathrm{RAM} 16.0 \mathrm{~GB}$, CPU. In order to verify the superiority of the proposed algorithm, different devices in different scenarios are selected for experiments. We also make comparison with the PDE method, NOBA method and the IKFCMLI method. The manual target domain segmentation of the original image is used as the gold standard segmentation image. The commonly used Jaccard Similarity (JS) and DICE Similarity Coefficient (DSC) are used as quantitative evaluation indexes. The specific formula is as follows. If the JS and DSC are close to 1 , the segmentation effect is better.

$$
\begin{array}{r}
J S=\frac{N\left(S_{g} \cap S_{m}\right)}{N\left(S_{g} \cup S_{m}\right)} . \\
D S C=\frac{2 N\left(S_{g} \cap S_{m}\right)}{N\left(S_{g}\right) \cup N\left(S_{m}\right)} .
\end{array}
$$

Where $S_{g}$ is the area of the gold standard segmentation. $S_{m}$ is the segmented area. $N(\cdot)$ is the number of pixels in the region.

The infrared image segmentation of power equipment is to extract the power equipment in the image. Fig. 2 is the original insulator infrared image in power system obtained by FLIR T630 infrared thermal imager. The gold standard segmentation is shown in Fig. 3.

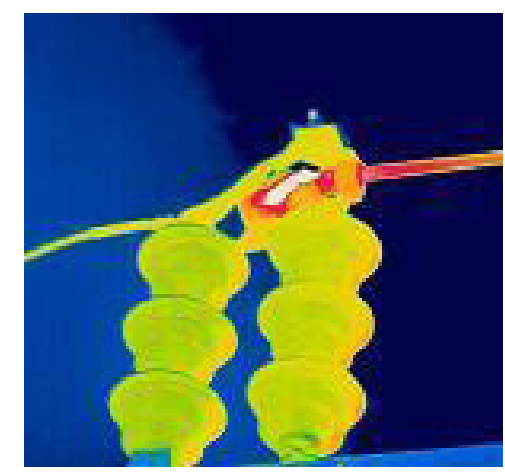

Fig. 2. The original insulator infrared image

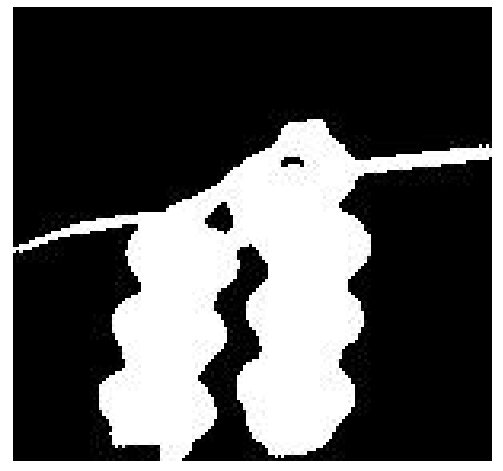

Fig. 3. The gold standard segmentation image 


\section{Experiment 1.}

Segmentation and comparison experiments are carried out on Fig. 2, the corresponding results are shown in figure 4. By comparing the segmentation effect with different methods in Fig. 4 with the gold standard segmentation pictures in Fig. 3, it can be seen that PDE and NOBA segmentation methods segment the white dots in the upper and middle region of the insulator into black dots in the background, while IKFCMLI and the proposed method in this paper are not obvious, the segmentation effects of both are similar. For other regions, the segmentation effects of the four methods are similar.

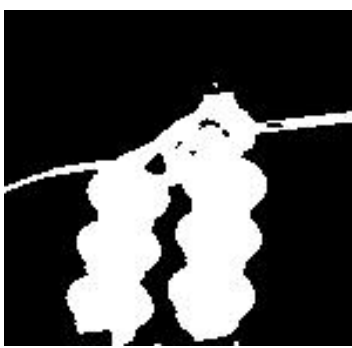

(a) PDE

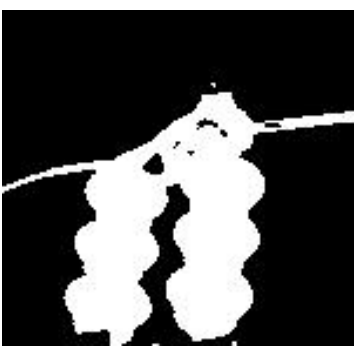

(b) NOBA

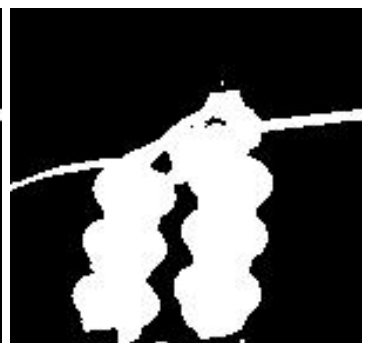

(c) IKFCMLI

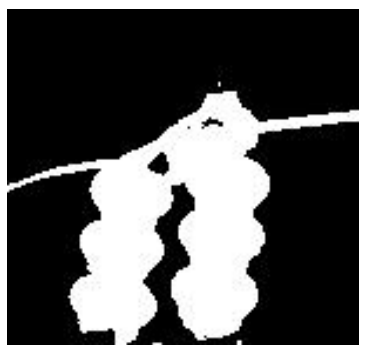

(d) Proposed method

Fig. 4. The segmentation image by different methods for the original image

Table 1 lists the quantitative evaluation results of the four segmentation methods in terms of JS and DSC. From Table 1, we can see that the proposed method in this paper has the best segmentation effect.

Table 1. Quantitative evaluation results of the four segmentation methods in terms of JS and DSC

\begin{tabular}{ccc}
\hline Method & JS & DSC \\
\hline PDE & 0.9612 & 0.9815 \\
NOBA & 0.9711 & 0.9824 \\
IKFCMLI & 0.9715 & 0.9853 \\
Proposed & $\mathbf{0 . 9 7 2 6}$ & $\mathbf{0 . 9 8 5 7}$ \\
\hline
\end{tabular}

\section{Experiment 2.}

We add 0.001 pepper and salt noise to the original insulator infrared image to make segmentation comparison. The image with added noise is shown in Fig. 5.

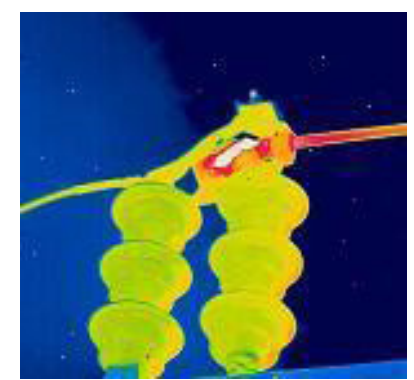

Fig. 5. The original insulator infrared image with 0.001 salt and pepper noise

Segmentation and comparison experiments are conducted on Fig. 5, the corresponding results are shown in Fig. 6. By comparing the segmentation effect of different methods in Fig. 6 with the gold standard segmentation image in Fig. 3, it can be intuitively seen that PDE and NOBA segment the white spots in the upper and middle area of the insulator into background black spots, and also segment some noise black spots in the left area of the background into white spots on the insulator. They filter out some added noise. IKFCMLI method missegments the background area noise black spots into insulator white spots, and it almost does not filter out the added noise. However, the proposed method does not have the obvious missegmentation phenomenon and almost eliminates all the added noise. 


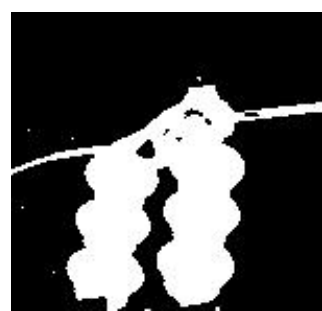

(a) PDE

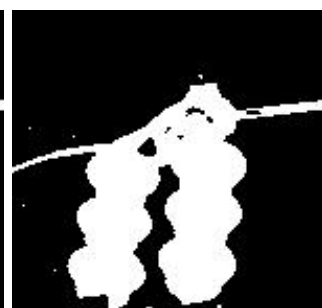

(b) NOBA

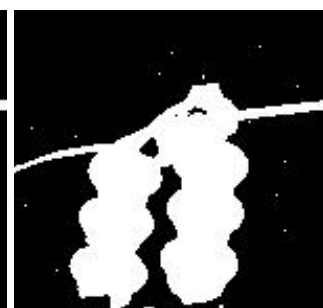

(c) IKFCMLI

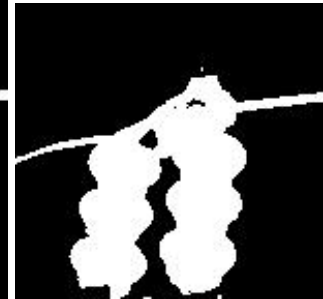

(d) Proposed method

Fig. 6. The segmentation image by different methods for the added noise image

Table 2 lists the quantitative evaluation results of the four segmentation methods in terms of JS and DSC under adding noise. From Table 2, we can see that the proposed method in this paper has the best segmentation effect, in which the values are close to 1 .

Table 2. Quantitative evaluation results of the four segmentation methods in terms of JS and DSC after adding noise

\begin{tabular}{ccc}
\hline Method & JS & DSC \\
\hline PDE & 0.9636 & 0.9826 \\
NOBA & 0.9705 & 0.9841 \\
IKFCMLI & 0.9711 & 0.9855 \\
Proposed & $\mathbf{0 . 9 7 2 7}$ & $\mathbf{0 . 9 8 6 7}$ \\
\hline
\end{tabular}

\section{Experiment 3.}

We also select other electric power equipment images to make comparison, the results are shown in Fig. 7. From Fig. 7 we can see that, there is no false detection for Voltage transformer with the proposed method. It can remove the complex background to achieve fine segmentation for Current transformer.

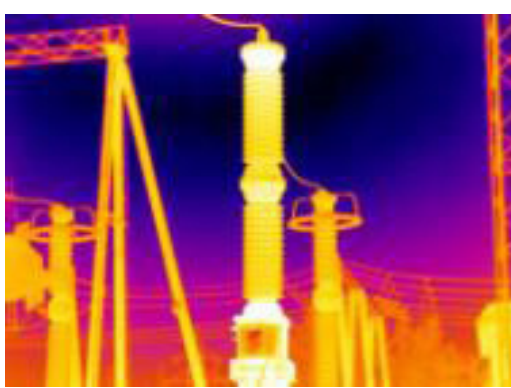

(a) Voltage transformer

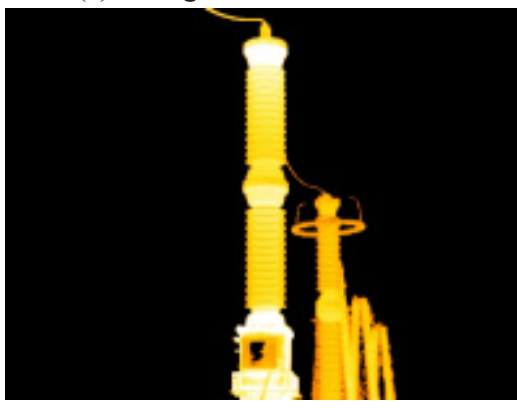

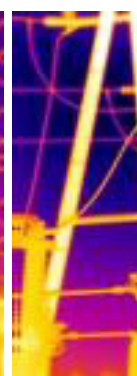

(b) Current transformer

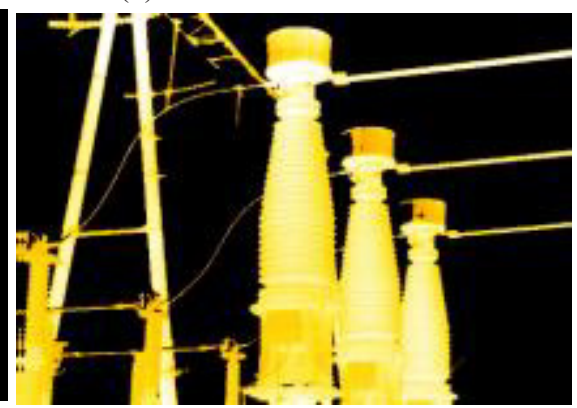

(a) PDE

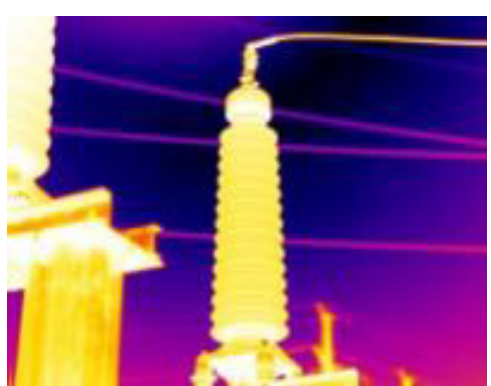

(c) Cable connector

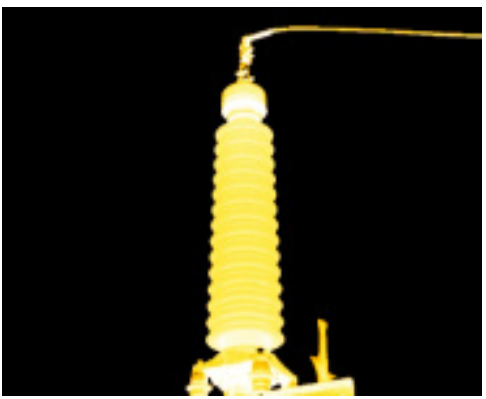



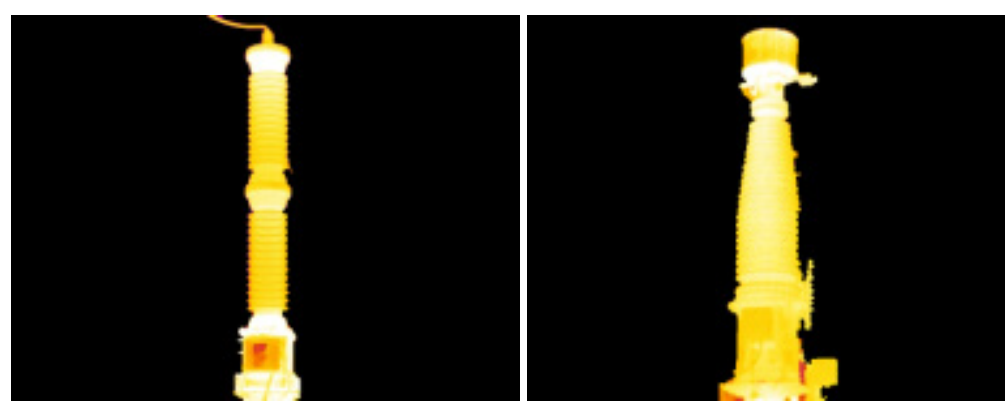

(b) NOBA
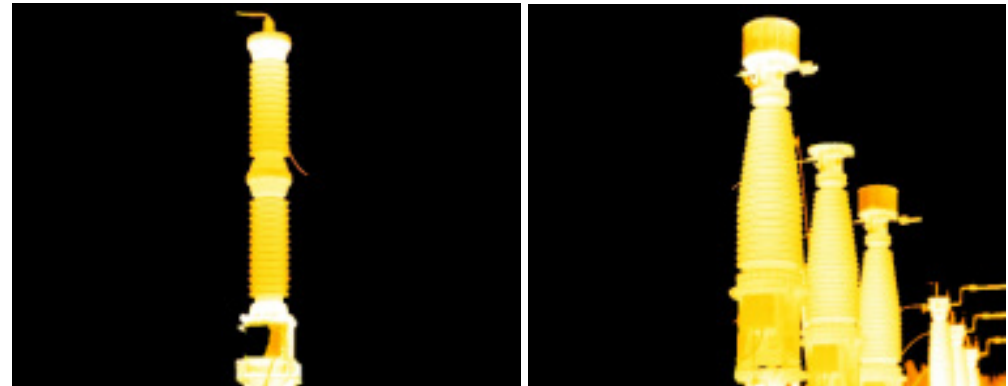

(c) IKFCMLI
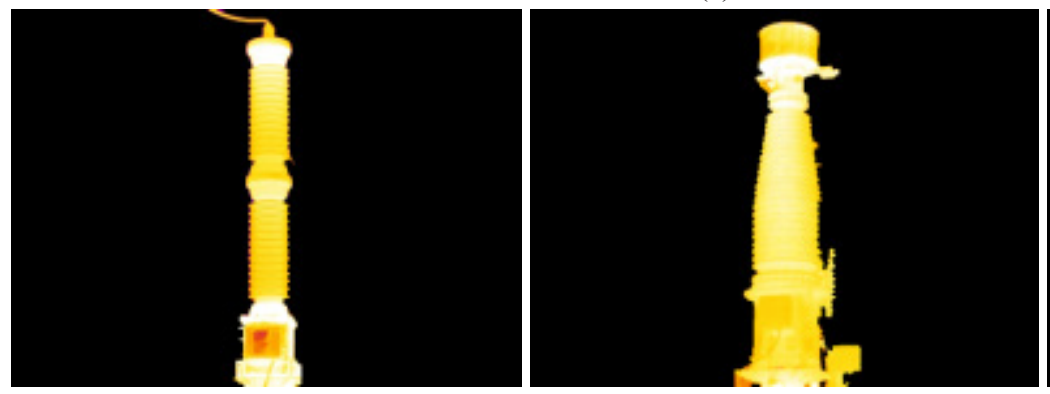

(d) Proposed
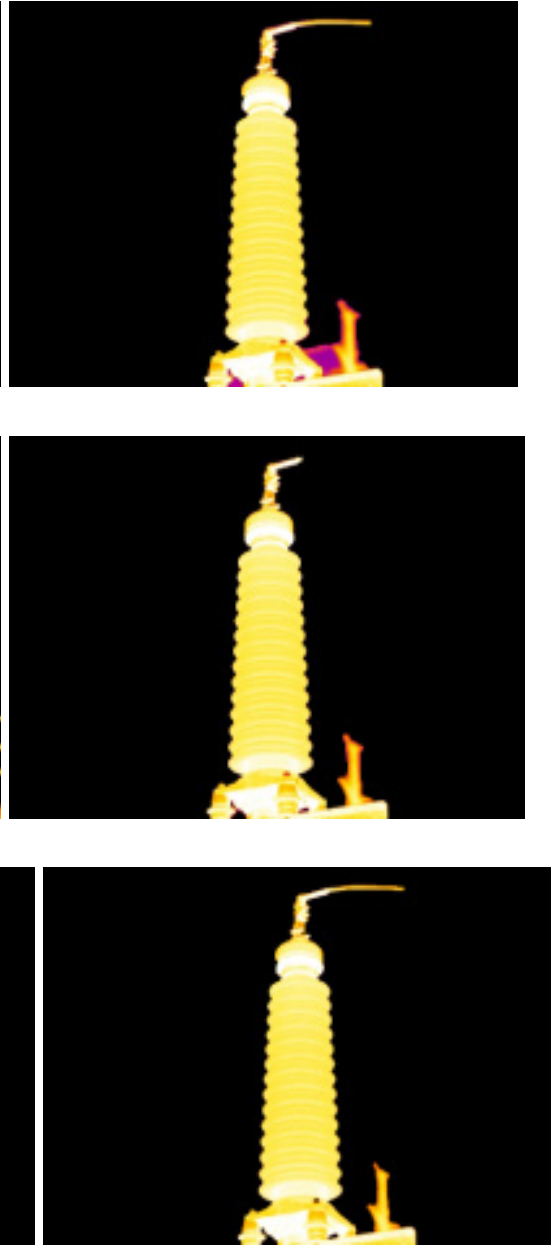

Fig. 7. The results with four methods for other electric power equipment images

Table 3 is the corresponding JS and DSC result. As can be seen from Table 3 and Fig. 7, the segmentation effect of the proposed algorithm is better than the other three methods. The OTSU threshold is used to pre-select the background super-pixel block, which eliminates the background located in the connected domain of the target equipment, highlights the structural characteristics of the equipment, and improves the segmentation effect of the target equipment. The LSC algorithm has a strong sensitivity to color, which makes full use of color information in the super-pixel segmentation stage to obtain a more reasonable segmentation. When the background pixel close to the target has high similarity to the target, the morphological operation can improve the problem of undersegmentation or over-segmentation. In terms of time consumption, the new algorithm in this paper also requires less time.

Table 3. Comparison with different methods

\begin{tabular}{cccccccccc}
\hline \multirow{2}{*}{ Method } & \multicolumn{3}{c}{ Voltage transformer } & \multicolumn{3}{c}{ Current transformer } & \multicolumn{3}{c}{ Cable connector } \\
\cline { 2 - 9 } & JS/\% & DSC/\% & Time/s & JS/\% & DSC/\% & Time/s & JS/\% & DSC/\% & Time/s \\
\cline { 2 - 9 } PDE & 97.66 & 98.64 & 25.54 & 97.75 & 97.19 & 58.26 & 96.21 & 96.65 & 29.28 \\
NOBA & 97.71 & 98.69 & 24.35 & 97.84 & 97.82 & 28.59 & 96.53 & 97.71 & 21.72 \\
IKFCMLI & 97.80 & 98.73 & 26.63 & 97.91 & 98.82 & 20.81 & 97.16 & 97.74 & 20.78 \\
Proposed & $\mathbf{9 7 . 8 4}$ & $\mathbf{9 8 . 7 9}$ & $\mathbf{1 4 . 0 1}$ & $\mathbf{9 7 . 9 6}$ & $\mathbf{9 8 . 8 8}$ & $\mathbf{1 9 . 9 2}$ & $\mathbf{9 7 . 8 5}$ & $\mathbf{9 8 . 8 1}$ & $\mathbf{1 4 . 0 2}$ \\
\hline
\end{tabular}

\section{Conclusions}

In this paper, a new method for infrared image segmentation of power equipment with complex background is proposed. The new method uses LSC super-pixel segmentation algorithm to segment the image. OTSU algorithm 
is combined with MSRM super-pixel merging algorithm. While merging the super-pixels with high similarity, the background contained in the target region is filtered to complete the rough extraction of the target equipment. The phenomenon of over-segmentation and under-segmentation is reduced effectively, and the target equipment is extracted completely. Morphological open/close algorithm is used to post-process the image to improve the segmentation accuracy of the target equipment. The experimental results show that the proposed algorithm can achieve higher segmentation accuracy in the infrared image segmentation of power equipment under complex background, and can effectively reduce the over-segmentation and under-segmentation rates. It highlights the structural characteristics of power equipment, and provides a foundation and convenience for subsequent structural region segmentation of power equipment and data analysis. In the future, we will research simple and high-efficiency deep learning methods to achieve better infrared image segmentation method for power equipment.

\section{Acknowledgement}

This work was supported by the Henan University of Higher Education Key Scientific Research Project. Name: Study on Evaluation Model of Online Education Learning Performance in Smart Campus (Project No. 21B880041).

\section{References}

[1] M. Li, H. Wang, Y. Yang, et al., Research and Implementation of a Power Equipment Infrared Diagnosis Algorithm, Gaoya Dianqi/High Voltage Apparatus 53(8)(2017) 224-229.

[2] C. Yu, L. Zeng, L. Zhang, Multi Point Fault Segmentation of Electrical Equipment Based on OTSU and Region Growth, Infrared Technology 40(10)(2018) 1008 -1012.

[3] Z. Huo, Q. Sun, F. Qi, et al., New Method of Fault Knowledge Acquisition of Electronic Equipment, Applied Mechanics \& Materials 496-500(2014) 931-934.

[4] Y. Wu, J. Yao, T. Li, P. Fu, W. Liao, M. Zhang, Application of Image Processing Techniques in Infrared Detection of Faulty Insulators, in: Proc. 6th Chinese Conference, CCPR 2014, 2014.

[5] H. Zou, F. Huang, Multi-target Localization for Infrared Images of Electrical Equipment Based on Improved FAsT-Match Algorithm, Proceedings of the CSEE, 37(2)(2017) 591-598.

[6] B. Yu, Y. Wan, S. Chen, et al., A density-similarity-factor-based segmentation method for infrared images of electric equipment, Infrared Technology 39(12)(2017) 1139-1143.

[7] J. Kimande, E. Mwangi, M. Gatari, Application of Infrared Thermography in Fault Detection and Preventive Maintenance in Three-Phase Distribution Transformers, International Journal of Computer Applications 174(12)(2021) 7-12.

[8] Q. Li, H. Cheng, Y. Zhou, et al., Road Vehicle Monitoring System Based on Intelligent Visual Internet of Things, Journal of Sensors (2015) 1-16.

[9] Y. Wang, N. Liu, H. Guo, et al., An engine-fault-diagnosis system based on sound intensity analysis and wavelet packet pre-processing neural network, Engineering Applications of Artificial Intelligence 94(2020).

[10]H. Liu, Y. Wang, H. Yao, A New Normalized-Cut Image Segmentation Algorithm Based on Watershed Transform, Applied Mechanics and Materials 235(2012) 45-48.

[11]M. Ayech, D. Ziou, Terahertz image segmentation using k-means clustering based on weighted feature learning and random pixel sampling, Neurocomputing 175(2016) 243-264.

[12]R. J. Al-Azawi, Q. S. Al-Jubouri and Y. A. Mohammed, Enhanced Algorithm of Superpixel Segmentation Using Simple Linear Iterative Clustering, in: Proc. 2019 12th International Conference on Developments in eSystems Engineering (DeSE), 2019.

[13]Z. Li and J. Chen, Superpixel segmentation using Linear Spectral Clustering, in: Proc. 2015 IEEE Conference on Computer Vision and Pattern Recognition (CVPR), 2015.

[14]Z. Wu, Interactive image segmentation by maximal similarity based region merging, Pattern Recognition 43(2)(2010) 445-456.

[15]Q. Chen, Modified two-dimensional Otsu image segmentation algorithm and fast realisation, Iet Image Processing 6(4) (2012) 426-433.

[16]Y. Hao, L. Dong, X. Liao, et al., A novel clustering algorithm based on mathematical morphology for wind power generation prediction, Renewable Energy 136(2019) 572-585.

[17]L. Nan, H. Rui, W. Zhuo, et al., Research on infrared image enhancement and segmentation of power equipment based on partial differential equation, Journal of visual communication \& image representation 64(2019) 102610.1-102610.8.

[18]W. Guo, H. Cui, et al., Research on Infrared Image Segmentation of Power Equipment Using Niblack Optimized by Bat Algorithm, DEStech Transactions on Engineering and Technology Research (2018). doi: 10.12783/dtetr/ pmsms2018/24925. 
Journal of Computers Vol. 33 No. 1, February 2022

[19]F. Hu, H. Chen and X. Wang, An Intuitionistic Kernel-Based Fuzzy C-Means Clustering Algorithm With Local Information for Power Equipment Image Segmentation, IEEE Access 8(2020) 4500-4514. 